Eur. J. Clin. Chem. Clin. Biochem.

Vol. 32, 1994, pp. 685-689

(c) 1994 Walter de Gruyter \& Co.

Berlin - New York

\title{
Neopterin Production and Tryptophan Degradation in Acute Lyme Neuroborreliosis Versus Late Lyme Encephalopathy
}

\author{
By T. Gasse ${ }^{1}$, C. Murr ${ }^{2}$, P. Meyersbach ${ }^{3}$, E. Schmutzhard ${ }^{1}$, H. Wachter ${ }^{2}$ and D. Fuchs ${ }^{2}$ \\ 1 Klinik fiir Neurologie \\ 2 Institut fiir Medizinische Chemie und Biochemie und Ludwig-Boltzmann Institut für AIDS-Forschung \\ 3 Zentralinstitut für Bluttransfusion \\ Universität Innsbruck, Innsbruck, Austria
}

(Received March 24/June 6, 1994)

Summary: Fourteen patients with Borrelia burgdorferi infection were investigated for possible abnormalities of tryptophan and neopterin metabolism. Four patients ( 2 were investigated before therapy, 2 when therapy had been already started) had acute Lyme neuroborreliosis, and 10 patients were investigated months to years after an acute infection. Increased concentrations of neopterin and of the tryptophan-degradation product, $L$-kynurenine, were detected in the cerebrospinal fluid of patients with acute Lyme neuroborreliosis; one patient presented with subnormal tryptophan. Similar but less marked changes were seen in the treated patients and in some of the patients with Lyme encephalopathy. No such abnormalities were seen in the serum of the patients. The data indicate a role of the immune system and particulary of endogenously formed cytokines, like interferon- $\gamma$ and tumour necrosis factor- $\alpha$, effecting tryptophan and neopterin metabolism in patients with acute Lyme neuroborreliosis.

\section{Introduction}

The tick-borne spirochaete, Borrelia burgdorferi, is the aetiological agent of Lyme borreliosis, a disease, which may also affect the nervous system. In the acute disseminated phase, meningitis, cranial neuritis and radiculoneuritis represent a typical triad of clinical manifestation. These symptoms respond rapidly to antibiotic therapy (1). Whether ongoing infection or other mechanisms cause chronic neurologic manifestations after adequate antibiotic therapy is still unclear.

Specific T- and B-lymphocyte responses have been demonstrated in humans (2) after infection with $B$. burgdorferi. Increased neopterin and $L=$ kynurenine concentrations in the cerebrospinal fluid of patients with Lyme neuroborreliosis have been described recently $(3,4)$. These data indicate a cellular immune response and intrathecal release of interferon- $\gamma$ in patients. Interferon- $\gamma$ strongly induces activation of GTP cyclohydrolase I and formation of neopterin in human monocytes/macrophages (5). Likewise, indoleamine $(2,3)$-dioxygenase is stimulated by interferon- $\gamma$ in human monocytes/macro- phages and in a variety of other cells and cell lines $(6-8)$. The induction of GTP cyclohydrolase I and indoleamine $(2,3)$-dioxygenase by interferon- $\gamma$ is enhanced by tumour necrosis factor- $\alpha$ (8).

In the present study, we compared tryptophan metabolism (i. e. changes in tryptophan and kynurenine concentrations) with neopterin levels in 4 patients with acute neuroborreliosis and in 10 patients months to years after the acute stage of the illness.

\section{Patients and Methods}

Patients

Fourteen serum and 13 cerebrospinal fluid samples were investigated from 4 patients with acute neuroborreliosis and 10 patients months to years after the acute stage of the illness. The patients ( $\delta / \%$ ratio $6 / 8$, median age 61 , range $28-70$ years at time of sample collection) were diagnosed and treated betiveen 1983 and 1992 at the Neurological Department, University of Innsbruck. Samples were taken from acute infection patients 1 and 2 before the commencement of antibiotic therapy, and from acute infection patients 3 and 4 after the commencement of antibiotic therapy (tab. 1). Ten patients were investigated months to years (median 41 , range 11 - 
106 months) after antibiotic therapy. These patients were part of an earlier evaluation of 20 patients with Lyme encephalopathy (9), who agreed to a spinal tap for cerebrospinal fluid collection. In one of these patients a spinal tap was not possible because of massive degenerative vertebral changes.

Concentrations of analytes were compared with normal ranges from earlier studies (tab. 2). As an additional control, serum from 20 healthy blood donors, 19-61 years of age, was included, because serum tryptophan and kynurenine concentrations in an earlier study (10) were obtained with a slightly different high-performance liquid chromatographic method.

\section{Methods}

Samples were stored at $-20^{\circ} \mathrm{C}$ until measurement. Neopterin concentrations were quantified by radioimmunoassay (IMMUtest Neopterin, Henning-Berlin, Berlin, Germany). Tryptophan and $L$ kynurenine concentrations were quantified using high-performance liquid chromatography with on-line deproteinization of serum and cerebrospinal fluid samples $(10,11)$. Separation was performed on reversed phase $\mathrm{C} 18$ material (LiChroCart, Merck, Darmstadt, Germany) injecting $100 \mu \mathrm{l}$ of sample via an advanced autosampling device (AASP, Varian, Palo Alto, California) using solid-phase extraction on cartridges (SCX, Varian) before liquid chromatography (10). Kynurenine was monitored by its ultraviolet absorption at 360 $\mathrm{nm}$, tryptophan by fluorescence detection at $365 \mathrm{~nm}$ emission and $285 \mathrm{~nm}$ excitation wavelengths. The limits of detection were 0.1 $\mu \mathrm{mol} / \mathrm{l}$ kynurenine (signal/noise ratio of $5 / 1$ ), $0.2 \mu \mathrm{mol} / \mathrm{l}$ tryptophan (signal/noise ratio of $5 / 1$ ) and $3 \mathrm{nmol} / \mathrm{l}$ neopterin.

For statistical evaluation we used non-parametric methods, namely the Mann-Whitney U-test for comparison between groups and the Spearman rank correlation analyses to analyse associations between analyte concentrations.

\section{Results}

In the diagnosis of Lyme neuroborreliosis, eight of the 14 patients showed the typical triad of neurological manifestations - meningitis, cranial neuritis (seven unior bilateral facial nerve palsy, one bilateral hypoacusis) and radiculoneuritis, four suffered from meningitis and radiculoneuritis, one from meningitis and cranial neuritis (right abducens paralysis) and one from cranial neuritis (bilateral abducens paralysis and hypoacusis, right facial nerve palsy) without meningitis. Six patients had a history of erythema migrans. Cerebrospinal fluid investigations displayed lymphocytic-predominant pleocytosis in 13 patients (median cell counts 110, range $8-357 \times 10^{6} / 1$ ) and elevated protein (median 1.29, range $0.70-5.58 \mathrm{~g} / \mathrm{l}$ ) in 12 of 13 patients. In nine patients intrathecal $B$. burgdorferi antibody production and/or $B$. burgdorferi specific oligoclonal antibodies were determined; one showed no sign of intrathecal antibody production (tab. 1). In this case neuroborreliosis was clinically diagnosed (typical triad of neurologic manifestations, arthralgias and immediate recovery after antibiotic treatment). In five patients, the ratio of intrathecal $B$. burgdorferi-specific antibody production versus serum titres was not determined: on case with radiculoneuritis and lymphocytic meningitis and a $B$. burgdorferi IgG-titre in cerebrospinal fluid $>1: 256$ (immunofluorescence test), one case with lymphocytic meningitis, radiculitic pain, bilateral facial palsy and positive $B$. burgdorferi serum IgG-titre $(1: 256)$ and IgM-titre $(1: 64)$ one year after onset of clinical symptoms (at first misdiagnosed as meningeal tuberculosis), one case with cranial neuritis 'without menigitis, serum IgG-titre $1: 64$ and IgM-titre $1: 128$, two cases with meningitis and radiculoneuritis and positive IgG in cerebrospinal fluid ( 1 case ELISA, 1 immunofluorescence test; titre was not determined). Overall duration of neurologic manifestations varied according to the time of diagnosis and treatment. Mean duration of involvement was 5 weeks ( 3 to 9 weeks); however, five patients had a much longer acute stage (3 to 12 months, mean 7.8 months). Nine patients (seen from 1990 to 1992) were treated with $2 \mathrm{~g}$ ceftriaxone for 14 days, four (seen from 1983 to 1987) received intravenous penicillin, 20 million units a day in a devided dose for ten days, and one was treated with tetracyclin, $2000 \mathrm{mg}$ a day, after developing an allergic reaction to penicillin.

At the time of sample collection for this evaluation, cerebrospinal fluid investigations of the four patients with acute illness (two before, two after onset of antibiotic treatment) displayed a lymphocytic pleocytosis and protein elevation (mean cell counts 86 , range 25-245 $\times 10^{6} / 1$; mean protein 0.87 , range $0.52-1.22 \mathrm{~g} / 1$ ). Eight of nine cerebrospinal fluid investigations out of the Lyme encephalopathy group were normal, and one showed a mild protein elevation $(0.59 \mathrm{~g} / \mathrm{l})$. Clinicāl follow-up of these ten patients revealed residual symptoms in four cases (mild right facial nerve palsy, mild radicular symptoms, mild radicular symptoms and right facial nerve palsy, bilateral abducens paralysis and hypoacusis).

In 20 healthy controls, serum concentrations of neopterin (mean \pm S. D.: $5.2 \pm 1.79 \mathrm{nmol} / \mathrm{l}$ ), tryptophan $(89.2 \pm 15.6 \mu \mathrm{mol} / \mathrm{l})$ and kynurenine $(2.1 \pm 0.49$ $\mu \mathrm{mol} / \mathrm{l})$ agreed well with the normal range found in earlier studies which, however, was determined with a slighly different high-performance liquid chromatography method. The kynurenine : tryptophan ratio was $24.4 \pm 7.4$.

In patients with acute Lyme neuroborreliosis a coincidental increase of the neopterin and kymurenine concentrations was observed, which was pronounced in cerebrospinal fluid but not in the serum of patients. All patients with acute Lyme neuroborreliosis and most patients with late Lyme encephalopathy had normal serum concentrations of neopterin (elevated in $2 / 10$ patients in the latter group) and kynurenine (elevated in $1 / 9$ patients in the latter group; tab. 2). Tryptophan concentrations in serum were below normal in $2 / 4$ patients with acute Lyme neuroborreliosis (both "untreated patients) and in 
Tab. 1 Clinical and diagnostic data in the acute stage of illness

\begin{tabular}{|c|c|c|c|c|c|c|c|c|c|c|c|c|c|c|}
\hline Patient & 1 & 2 & 3 & 4 & 5 & 6 & 7 & 8 & 9 & 10 & 11 & 12 & 13 & 14 \\
\hline Age (a) & 64 & 35 & 28 & 35 & 62 & 64 & 66 & 48 & 38 & 62 & 64 & 29 & 64 & 54 \\
\hline Sex & q & q & 우 & $\delta$ & q & $\delta$ & 우 & $\sigma$ & 우 & $q$ & 우 & $\delta$ & $\delta$ & $\sigma$ \\
\hline Durations of symptoms (weeks) & 5 & 5 & 48 & 3 & 9 & 12 & 48 & 4 & 4 & 5 & 28 & 5 & 20 & 5 \\
\hline Meningitis, cranial neuritis and radiculoneuritis & + & + & + & + & + & - & + & + & - & + & - & - & - & - \\
\hline Meningitis and cranial neuritis & - & - & - & - & - & - & - & - & + & - & $*$ & - & - & - \\
\hline Meningitis and radiculoneuritis & - & - & - & - & - & + & - & - & - & - & - & + & + & + \\
\hline Erythema migrans & + & - & - & + & + & - & - & - & + & + & - & + & - & - \\
\hline $\begin{array}{l}\text { Intrathecal Borrelia burgdorferi } \\
\text { antibody production }\end{array}$ & - & - & + & + & - & nd & nd & + & + & + & nd & + & nd & nd \\
\hline $\begin{array}{l}\text { Borrelia burgdorferi } \\
\quad \text { specific oligoclonal antibody production }\end{array}$ & + & nd & + & + & + & nd & nd & nd & nd & nd & nd & nd & nd & nd \\
\hline Cerebrospinal fluid cell counts $\left(10^{6} / 1\right)$ & 245 & 25 & 309 & 110 & 187 & 16 & 357 & 56 & 13 & 192 & 1 & 193 & 8 & 51 \\
\hline Cerebrospinal fluid protein $(\mathrm{g} / \mathrm{l})$ & 1.2 & 0.7 & 5.6 & 1.3 & 1.0 & 1.3 & 1.6 & 1.8 & 0.4 & 1.7 & 0.5 & 0.8 & 1.3 & 2.0 \\
\hline
\end{tabular}

$+=$ present $-=$ absent nd $=$ not determined

* = case 11 suffered from cranial neuritis without meningitis

Tab. 2 Results of measurements in serum and cerebrospinal fluid and of patients months to years after antibiotic therapy (patients (CSF) of patients with acute Lyme neuroborreliosis (patients 1-4) 5-14)

\begin{tabular}{|c|c|c|c|c|c|c|c|}
\hline & \multicolumn{2}{|c|}{$\begin{array}{l}\text { Neopterin } \\
(\mathrm{nmol} / \mathrm{l})\end{array}$} & \multicolumn{2}{|c|}{$\begin{array}{l}\text { Tryptophan } \\
(\mu \mathrm{mol} / \Omega)\end{array}$} & \multicolumn{2}{|c|}{$\begin{array}{l}\text { Kynurenine } \\
(\mu \mathrm{mol} / \mathrm{l})\end{array}$} & \multirow[t]{2}{*}{$\begin{array}{l}\text { Time from acute } \\
\text { disease (months) }\end{array}$} \\
\hline & Serum & CSF & Serum & CSF & Serum & $\mathrm{CSF}$ & \\
\hline $\begin{array}{l}\text { Reference range } \\
\text { (Reference) }\end{array}$ & $\begin{array}{l}<8.8 \\
(5)\end{array}$ & $\begin{array}{l}<6.0 \\
(12)\end{array}$ & $>57.0$ & $\begin{array}{l}>1.0 \\
(4)\end{array}$ & $<3.1$ & $\begin{array}{l}<0.1 \\
(4)\end{array}$ & \\
\hline
\end{tabular}

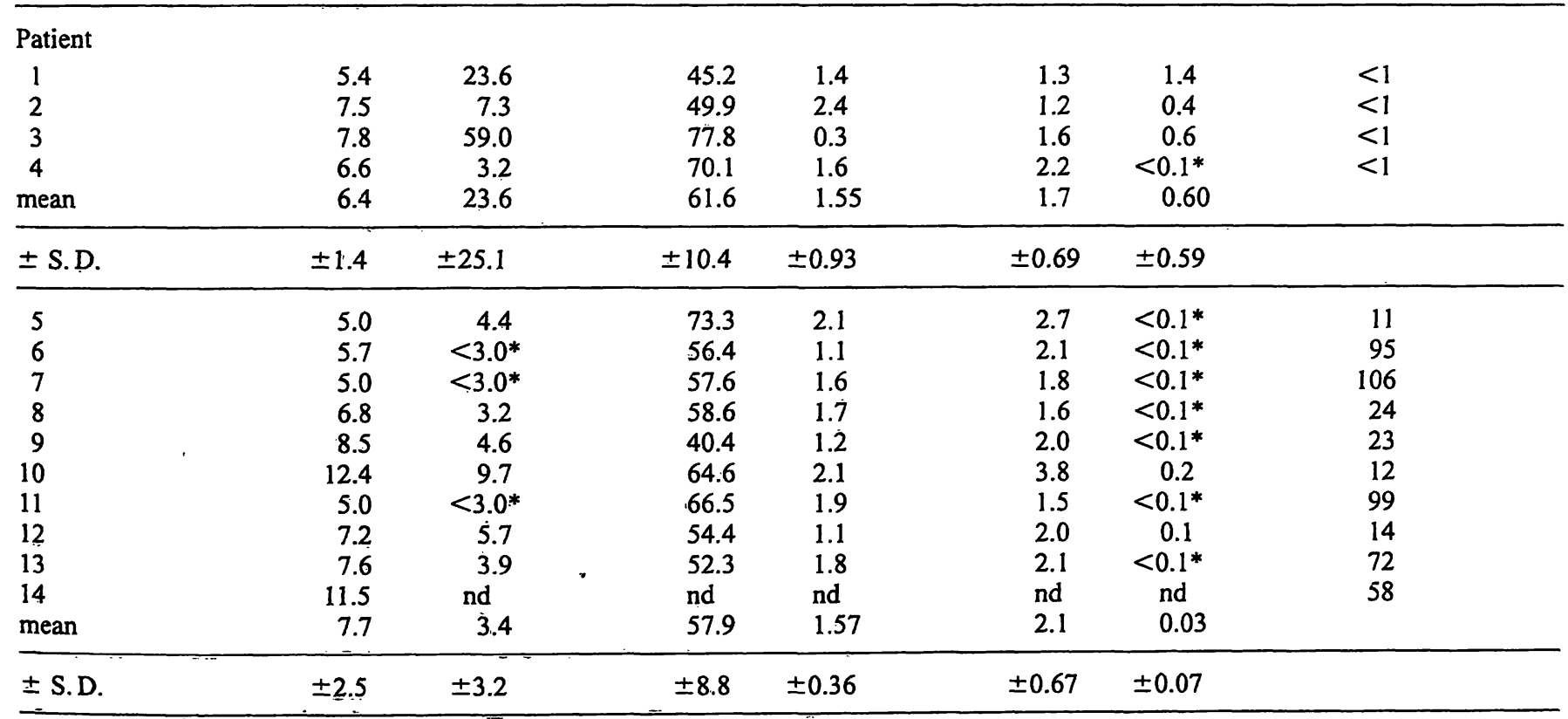

nd $=$ not determined

${ }^{*}=$ below detection limit; for statistical evaluation these values were calculated as zero

4/9 patients with late Lyme encephalopathy. In contrast, in cerebrospinal fluid samples, $3 / 4$ patients with acute Lyme neuroborreliosis had increased neopterin concen- trations ( 1 treated patient had neopterin within the normal range). Concentrations of kynurenine behaved similarly ( 3 patients with elevated kynurenine concentra- 
tions, 1 treated patient with normal kynurenine). The patient with the highest neopterin concentration showed a subnormal concentration of tryptophan, despite this fact the kynurenine concentration was high. Taking into consideration the low tryptophan concentration in cerebrospinal fluid, the relative amount of kynurenine was by far the highest in patient 3 (kynurenine per tryptophan ratio $=2000$ ), followed by patient 1 (ratio: 1000), which mirrors exactly the magnitude of neopterin levels in cerebrospinal fluid of the same patients (tab. 2).

A significant correlation existed between neopterin concentrations and mononuclear cell count in cerebrospinal fluid ( $r s=0.625, p=0.030$ ). Similar and significant correlations were found between the concentrations of neopterin and kynurenine and the kynurenine : tryptophan ratio; the correlations were strongest between neopterin and kynurenine in cerebrospinal fluid: $\mathrm{rs}=0.871, \mathrm{p}=0.0031$ in all patients; $\mathrm{rs}=0.743$, $p=0.036$ in patients with late Lyme encephalopathy only (fig: '1). Although most patients with late Lyme encephalopathy had normal neopterin (1/9 elevated) and kynurenine ( $2 / 9$ detectable $\triangleq$ elevated), and all had normal tryptophan concentrations in the cerebrospinal fluid, there is still a similar degree of correlation between the concentrations of kynurenine and neopterin (fig. 1).

Concentration changes in patient sera were only marginal compared with those in cerebrospinal fluid, but average serum tryptophan concentrations were significantly decreased in patients $(n=13: 59.0 \pm 11.0$ $\mu \mathrm{mol} / \mathrm{l})$ compared with the group of healthy controls $(\mathrm{n}=20: 89.2 \pm 15.6 \mu \mathrm{mol} / \mathrm{l} ; \mathrm{U}=4.33, \mathrm{p}<0.0001)$.

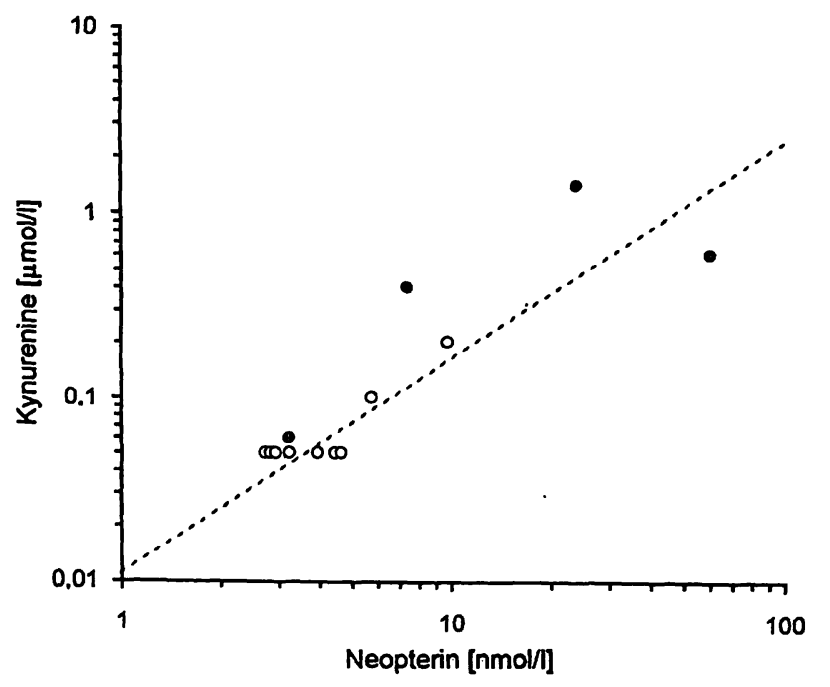

Fig. 1 Correlation between concentrations of neopterin and $\mathbf{k y -}$ nurenine in cerebrospinal fluid of patients with acute neuroborreliosis (filled circles) and late Lyme encephalopathy (open circles). Spearman rank correlation coefficients: $\mathrm{rs}=0.871, \mathrm{p}=0.0026$ (all patients, $\mathrm{n}=13$ ), $\mathrm{rs}=0.743, \mathrm{p}=0.036$ (only patients with late Lyme encephalopathy, $n=9$ ). Note logarithmic scale of analyte concentrations.
Neopterin was higher in patients $(n=14: 7.4 \pm 2.2$ $\mathrm{nmol} / \mathrm{l})$ than in the controls $(\mathrm{n}=20: 5.2 \pm 1.8 \mathrm{nmol} / \mathrm{l}$; $\mathrm{U}=3.13, \mathrm{p}=0.0017$ ). Interestingly, a correlation existed between serum and cerebrospinal fluid tryptophan concentrations ( $r s=0.714, p=0.043$ ) in the subgroup of patients with late Lyme encephalopathy. Cerebrospinal fluid concentrations of neopterin $(U=2.10$; $p<0.05)$ and kynurenine $(U=2.11 ; p<0.05)$ were higher in patients with acute Lyme neuroborreliosis compared with those with late Lyme encephalopathy, whereas tryptophan concentrations did not differ. In serum, no differences were found between patients with acute Lyme neuroborreliosis and patients with late Lyme encephalopathy.

\section{Discussion}

The data confirm and extend earlier results obtained in patients with $B$. burdorferi infection $(3,4)$ and are consistent with the view that degradation of tryptophan due to immune activation was the reason for the accumulation of $L$-kynurenine. Increased concentrations of neopterin in cerebrospinal fluid indicate activation of immunocompetent cells in the brain and indirectly confirm the endogenous formation of cytokines, such as interferon- $\gamma$ and tumour necrosis factor- $\alpha$, in the patients, because large amounts of neopterin are released from human monocytes/macrophages upon activation with interferon- $\gamma$ in vitro. Our data also demonstrate that significant degradation of tryptophan occurs in the cerebrospinal fluid of patients with acute Lyme neuroborreliosis. Thus, the increase of the tryptophan metabolite, kynurenine, correlates with the increase of neopterin. Importantly, kynurenine was even higher in patient 3 , who had an extremely low tryptophan concentration in the cerebrospinal fluid (tab. 2). This observation can be explained by the strong activation of indoleamine $(2,3)$ dioxygenase, as indicated by the very high kynurenine : tryptophan ratio of 2000 in the same patient. Interestingly, similar associations between the formation of neopterin and kynurenine have also been demonstrated in patients suffering from multiple sclerosis (13). Changes of tryptophan metabolism possibly play a role in the pathogenesis of neurological symptoms in patients. On the one hand, a decrease of tryptophan may disturb serotonin metabolism (4); on the other hand neurotoxic products of tryptophan degradation, like kynurenic acid or quinolinic acid, may have a role (14). One of our patients showed a severely decreased tryptophan level in cerebrospinal fluid, and serum tryptophan levels tended to decrease in several patients of both groups, even when there was no detectable increase of tryptophan metabolites in serum. It appears that, in the chronic situation, degradation of tryptophan in cerebro- 
spinal fluid may also contribute to the deficiency of tryptophan in serum by causing a compensatory flux of tryptophan into the brain. Interestingly, a significant association between serum and cerebrospinal fluid tryptophan levels was found in the patients with late Lyme encephalopathy ( $r s=0.714, p=0.05$ ).

When comparing late Lyme encephalopathy with acute Lyme neuroborreliosis, it is evident that metabolic changes in cerebrospinal fluid are much more pronounced in the latter group of patients. However, similar correlations existed between the changes of tryptophan metabolism and neopterin in late Lyme encephalopathy, which suggests a low-level, on-going infection, at least in some patients. This would lead to low-grade chronic

\section{References}

1. Pachner, A. R. \& Steere, A. C. (1985) The triad of neurologic manifestations of Lyme disease. Neurology 35, 47-53.

2. Dattwyler, R. J., Volkman, D. J., Luft, B. J., Halperin, J., Thomas, J. \& Golightly, M. G. (1988) Seronegative Lyme disease: Dissociation of specific T- and B-lymphocyte response to Borrelia burgdorferi. New Engl. J. Med. 319, 1441-1446.

3. Dotevall, L., Fuchs, D., Reibnegger, G., Wachter, H. \& Hagberg, L. (1990) Cerebrospinal fluid and serum neopterin levels in patients with Lyme neuroborreliosis. Infection 18, 210214.

4. Fuchs, D., Dotevall, L., Hagberg, L., Werner, E. R. \& Wachter, H. (1991) Kynurenine in cerebrospinal fluid of patients with Lyme neuroborreliosis. Immunol. Infect. Dis. 1, 271-274.

5. Fuchs, D., Hausen, A., Reibnegger, G., Werner, E. R., Dierich, M. P. \& Wachter, H. (1988) Neopterin as a marker for activated cell-mediated immunity: Application in HIV infection. Immunol. Today $9,150-155$.

6. Byme, G., Lehmann, L. K., Kirschbaum, J. G., Borden, E. C., Lee, C. M. \& Brown, R. R. (1986) Induction of tryptophan degradation in vitro and in vivo: A gamma interferon stimulated activity. J. Interferon Res. 6, 389-398.

7. Werner, E. R., Bitterlich, G., Fuchs, D., Hausen, A., Reibnegger, G., Szabo, G., Dierich, M. P. \& Wachter, H. (1987) Human macrophages degrade tryptophan upon induction by interferon gamma. Life Sci. 42, 310-316.

8. Werner-Felmayer, G., Werner, E. R., Fuchs, D., Hausen, A., Reibnegger, G. \& Wachter, H. (1989) Characteristics of interferon induced tryptophan metabölism in human cells in vitro. Biochim. Biophys. Acta 1012, 140-147. immune activation, with increased degradation of tryptophan. In our patients with late Lyme encephalopathy, however, we found no correlation between biochemical changes and specific clinical symptoms, such as chronic neurological manifestations. From this observation, it appears that irreversible damage of brain tissue, particularly during the acute phase of Lyme neuroborreliosis, is the major determinant of late symptoms in patients. Further studies are needed to clarify this point.

\section{Acknowledgement}

This study was supported by the Austrian granting body "Zur Förderung wissenschaftlicher Forschung", P 9257.

9. Benke, Th., Gasse, Th., Hittmair-Delazer, M. \& Schmutzhard, E. (1994) Lyme encephalopathy: A study of longterm neuropsychological deficits. Acta Neurol. Scand. (in press).

10. Werner, E. R., Fuchs, D., Hausen, A., Reibnegger, G. \& Wachter, H. (1987) Simultaneous determination of neopterin and creatinine in serum with solid-phase extraction and on-line elution liquid chromatography. Clin. Chem. 33, 2028-2033.

11. Fuchs, D., Möller, A. A., Reibnegger, G., Stöckle, E., Werner, E. R. \& Wachter, H. (1990) Decreased serum tyroptophan in patients with HIV infection correlates with increased serum neopterin and with neurologic/psychiatric symptoms. J. Acquir. Immune Defic. Syndr. 3, 873-876.

12. Hagberg, L., Dotevall, L., Norkrans, G., Larsson, M., Wachter, H. \& Fuchs, D. (1993) Cerebrospinal fluid neopterin concentrations in central nervous system infection. J. Infect. Dis. 168 , 1285-1288.

13. Ott, M., Demisch, L., Engelhardt, W. \& Fischer, P.-A. (1993) Interleukin-2, soluble interleukin-2-receptor, neopterin, L-tryptophan and $\beta_{2}$-microglobulin levels in cerebrospinal fluid and serum of patients with relapsing-remitting or chronic-progressive multiple sclerosis. J. Neurol. 241, 108-114.

14. Halperin, J. J. \& Heyes, M. P. (1992) Neuroactive kynurenines in Lyme borreliosis. Neurology 42, 43-50.

Univ.-Doz. Dr. Dietmar Fuchs

Institut für Medizinische Chemie und Biochemie

Universität Innsbruck

Fritz-Pregl-Straße 3

A-6020 Innsbruck

Austria 


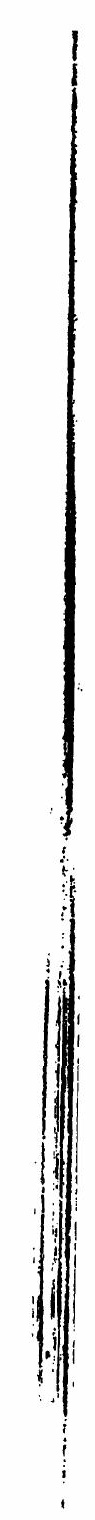

